\title{
Study of Integrated Spectra of Four Globular Clusters in M31
}

\author{
M. I. Maricheva ${ }^{1, *}$ \\ ${ }^{1}$ Special Astrophysical Observatory, Russian Academy of Sciences, Nizhnii Arkhyz, 369167 Russia
}

The results of determining the metallicity, age, helium mass fraction $(\mathrm{Y})$ and abundances of the elements C, N, Mg, Ca, Mn, Ti and Cr by moderate resolution spectra for four globular clusters in the galaxy M31: Bol 6, Bol 20, Bol 45 and Bol 50 are presented. The chemical composition for Bol 20 and Bol 50, and Y for four clusters are determined for the first time. The spectra of the studied objects were obtained with the 6-meter telescope of the SAO RAS in 2020. All the clusters under study turned out to be older than 11 Gyrs. The determined metallicities $[\mathrm{Fe} / \mathrm{H}]$ are in the range from -1.1 to -0.75 dex. They are lower than the metallicity of stars of the M31 halo at a given distance from the galactic center $\left(\mathrm{R}_{M 31}\right.$ $<10 \mathrm{kpc})$. The abundances of the elements of the $\alpha$-process $[\alpha / \mathrm{Fe}]=([\mathrm{O} / \mathrm{Fe}]+[\mathrm{Mg} / \mathrm{Fe}]+$ $[\mathrm{Ca} / \mathrm{Fe}]) / 3$ of the four clusters correspond to those of the stars of the inner halo of M31.

\section{INTRODUCTION}

Studying systems of globular clusters of galaxies provides a clue to the evolution and processes of stellar formation in them. M31 is the closest spiral galaxy to the Milky Way. The study of its star clusters is of great interest. The system of globular clusters in M31 is one of the most studied (see, for example, Caldwell et al. 2016; Mackey et al. 2019; McConnachie et al. 2018, and references therein). However, deep color-magnitude diagrams (CMD) of globular clusters in M31, reaching the Main Sequence (MS) turnoff point, have not yet been obtained using observations either with Hubble Space Telescope (HST), nor with the largest ground-based telescopes due to the distance of M31 (according to the conclusions of Riess et al.
(2016), the distance to M31 is $\mathrm{D}_{\mathrm{M} 31}=0.745 \pm$ $0.028 \mathrm{Mpc}$ ) and a high density of stars in globular clusters, which makes it difficult to perform photometry (see the paper by Federici et al. 2012, and references therein). Literature sources mainly present the integral characteristics of globular clusters in M31 (colors, spectra) in order to determine their age and metallicity by comparison with models of simple stellar populations (see, for example, Caldwell et al. 2009; Cezario et al. 2013; Fan et al. 2016; Wang et al. 2019). High-resolution integrated-light spectroscopy $(R>20$ 000) of bright clusters in M31 was performed by Colucci et al. (2014) and Sakari et al. (2016). As noted by Sakari et al. (2014), the study of integrated-light spectra of clusters of high spectral resolution often does not give great advantages compared to moderateresolution spectroscopy $(\mathrm{R}<5000)$, since the ac-

* Electronic address: marichevar@gmail.com 
cumulation of a large signal in the spectra is necessary for the confident determination of the chemical abundances, which is impossible at high resolution, since it requires significant observational time. Even when determining the iron abundance, which has the largest number of lines in the spectra compared to other elements, measurement errors in high-resolution spectra are of the order of $0.1-0.4$ dex.

This work is devoted to the study of the integrated-light spectra of moderate resolution of four bright globular clusters in M31: Bol 6, Bol 20, Bol 45 and Bol 50. All the studied objects, except Bol 50, were first discovered by Hubble (Hubble 1932) as likely candidates for globular clusters. Bol 50 was discovered by Baade and was first mentioned by Seyfert and Nassau (1945). All these objects are included in the catalog by Galleti et al. (2004). The main properties of clusters are listed in Table 1: the first column contains the identifiers from Galleti et al. (2004), then-right ascension and declination, visible stellar magnitudes in the $\mathrm{V}$ band of the Johnson- Cousins photometric system, colour excesses $\mathrm{E}(\mathrm{B}-\mathrm{V})^{1}$, radial velocities, projected distances from the center of M31, halflight radii. Clusters have close celestial coordinates, but their radial velocities are different. The objects are located in the sky area between

\footnotetext{
${ }^{1} \mathrm{E}(\mathrm{B}-\mathrm{V})=\mathrm{A}_{B}-\mathrm{A}_{V}$, where $\mathrm{A}_{B}$ and $\mathrm{A}_{V}$ are the extinction in stellar magnitudes in the $\mathrm{B}$ - and $\mathrm{V}$-bands, respectively.According to Schlegel et al. (1998), $\mathrm{A}_{V}=$ $3.315 \mathrm{E}(\mathrm{B}-\mathrm{V}), \mathrm{A}_{I}=1.940 \mathrm{E}(\mathrm{B}-\mathrm{V})$.
}

M31 and its satellite - the dwarf elliptical galaxy NGC205 (Fig. 1, the DSS digital sky survey frame $^{2}$ ), located at a distance of $0.824 \mathrm{Mpc}$ from the Sun, according to McConnachie et al. (2005).

\section{OBSERVATIONAL DATA AND} REDUCTION OF THE SPECTRA

The integrated-light spectra of all four objects were acquired with the $6-\mathrm{m}$ Telescope of the SAO RAS on September 19, 2020, under the program of M.E. Sharina "Properties of stellar populations of extragalactic globular clusters". The observations were carried out using the primary focus focal reducer SCORPIO-1 (Afanasiev and Moiseev 2005) in the long-slit spectroscopy mode. The VPHG1200B grism was used, which provides the spectral range 3600-5400 $\AA$ and a resolution (full width at half maximum of a single spectral line) of the order of FWHM $\sim 5.5 \AA$. The slit width of $1^{\prime \prime}$ was chosen. The observation log is presented in Table 2, in which the total exposure times, the ratio $\mathrm{S} / \mathrm{N}$ (signal-to-noise) per pixel at the wavelength obtained in the resulting total one-dimensional spectra, and the seeing are indicated after the name of the object.

The reduction of long-slit spectra was performed using MIDAS (Banse et al.1983) and IRAF (Tody 1993) software packages. The frames were cleared of cosmic particles and linearized by the spectra of the He- Ne-Ar lamp

\footnotetext{
${ }^{2}$ http://archive.stsci.edu/cgi-bin/dss_form
} 
Table 1. Main characteristics of the studied clusters

\begin{tabular}{c|c|c|c|c|c|c}
\hline Object & $\begin{array}{c}\text { RA DEC (2000) } \\
\text { hh:mm:ss gr:mm:ss }\end{array}$ & $\begin{array}{c}\mathrm{V} \\
\mathrm{mag}\end{array}$ & $\begin{array}{c}\mathrm{E}(\mathrm{B}-\mathrm{V})^{a} \\
\mathrm{mag}\end{array}$ & $\begin{array}{c}\mathrm{Vel}^{a} \\
\mathrm{~km} \mathrm{~s}^{-1}\end{array}$ & $\begin{array}{c}\mathrm{R}_{M 31}^{b} \\
\mathrm{kpc}\end{array}$ & $\begin{array}{c}\mathrm{R}_{h}^{c} \\
\mathrm{pc}\end{array}$ \\
\hline Bol6 & $00: 40: 26.47+41: 27: 26.6$ & 15.97 & 0.17 & $-232.4 \pm 6$ & 6.3 & 1.86 \\
Bol20 & $00: 40: 55.26+41: 41: 25.3$ & 16.13 & 0.11 & $-345.4 \pm 5$ & 7.3 & 3.17 \\
Bol45 & $00: 41: 43.11+41: 34: 20.1$ & 15.14 & 0.18 & $-419.4 \pm 6$ & 4.8 & 2.85 \\
Bol50 & $00: 41: 46.27+41: 32: 18.4$ & 16.79 & 0.25 & $-109.5 \pm 6$ & 4.4 & - \\
\hline
\end{tabular}

a - Caldwell et al. (2011); b - Caldwell et al. (2016); c - Barmby et al. (2007)

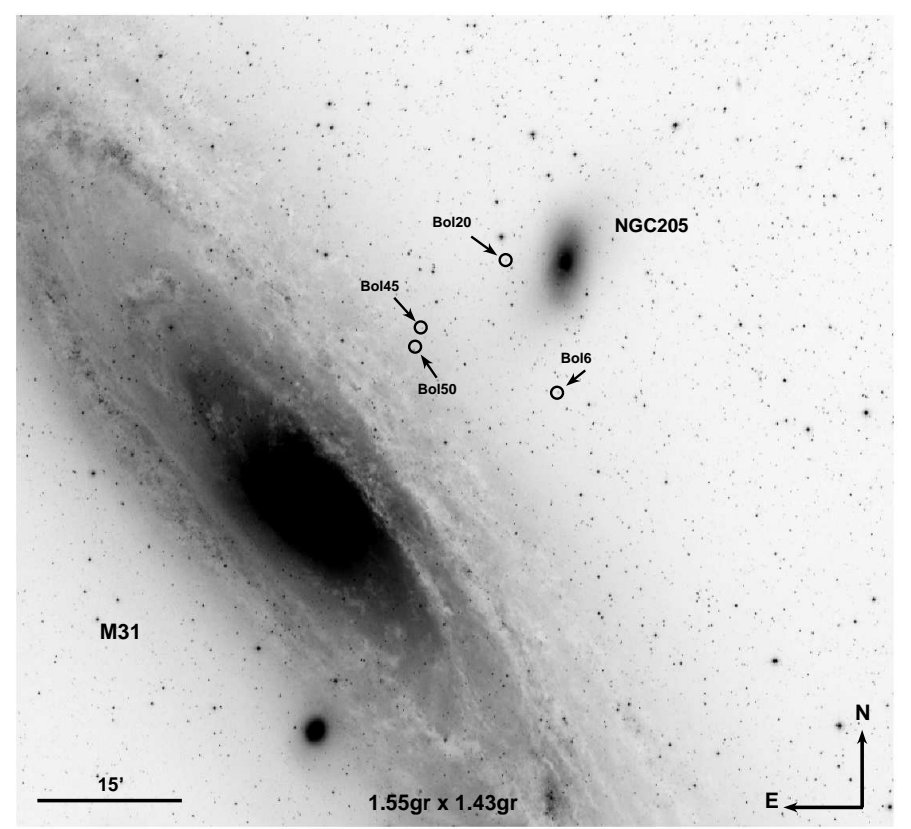

Figure 1. The position of the studied clusters in the projection onto the celestial sphere in the frame of the DSS digital sky survey.

using MIDAS. The dispersion relation ensured the accuracy of calibration of wavelengths of the order of $0.16 \AA$. The sky background was subtracted in IRAF using the background procedure. One-dimensional spectra were extracted in IRAF using the apsum procedure with correction of the curvature of the spectrum along the dispersion.
Table 2. Observation $\log$

\begin{tabular}{c|c|c|c}
\hline Object & $\mathrm{t}_{\exp }$ & $\mathrm{S} / \mathrm{N}$ & Seeing \\
& $\mathrm{s}$ & $(4500 \AA)$ & \\
\hline Bol6 & $3 \times 900$ & 75 & $2.5^{\prime \prime}$ \\
Bol20 & $3 \times 900$ & 100 & $1.5^{\prime \prime}$ \\
Bol45 & $3 \times 600$ & 121 & $2.5^{\prime \prime}$ \\
Bol50 & $3 \times 900$ & 56 & $1.5^{\prime \prime}$ \\
\hline
\end{tabular}

To increase the $\mathrm{S} / \mathrm{N}$ ratio, the obtained one responding integral spectra of the clusters Bol dimensional spectra were summed with the cor- 6, Bol 20, Bol 45 and Bol 50 from the observa- 
tion archive of the Hectospec spectrograph of the 6.5-m MMT telescope (Fabricant et al. 2005), which were taken with a 270 grooves $/ \mathrm{mm}$ grating with a dispersion of $1.21 \AA$ / pixel in the spectral range 3650-9200 $\AA$ and they have the resolution FWHM $\sim 5 \AA$. These spectra were used by Caldwell et al. $(2009,2011)$ to study the age, metallicity, and kinematic characteristics of clusters in M31. When summing up with our spectra, they were reduced to the same spectral resolution depending on the wavelength. As a result, the $\mathrm{S} / \mathrm{N}$ ratio in the obtained total spectra of clusters varies from 100 for Bol 50 to 280 for Bol 20 at a wavelength of $4500 \AA$. Determination of the dependence of the spectral resolution on the wavelength and smoothing of the spectra to the required resolution was performed taking into account the line spread function (LSF) of the spectrograph using the UlySS software package of the University of Lyon (Koleva et al.2008, 2009). UlySS is an open source package that allows you to compare the observed and model spectra by nonlinear minimization of their difference by the least squares method. The UlySS program also performs spectrum normalization by introducing a multiplicative polynomial to scale the model spectrum. The UlySS web page provides an example of how to build $\mathrm{LSFF}^{3}$. Examples of the LSF calculated in this work for observations with SCORPIO-I and Hectospec spec-

\footnotetext{
${ }^{3}$ http://ulyss.univ-lyon1.fr/tuto_base.html
}

trographs are given on the SAO RAS website ${ }^{4}$.

3. METHOD FOR DETERMINING THE AGE, HELIUM MASS FRACTION AND
CHEMICAL COMPOSITION

The method is developed and described in detail by Sharina et al. (2020) (see also the references therein). It is used to determine the age, the helium mass fraction $(\mathrm{Y})$ and the chemical composition of old globular clusters of our and other galaxies (see, for example, Sharina et al. 2020, 2018).

Within the framework of this method, the synthetic integral spectra of cluster stars are calculated according to the atmospheric parameters set up by the isochrones of stellar evolution. The spectra of individual stars are calculated using the CLUSTER program (Sharina et al. (2020) and references therein) under the approximation of local thermodynamic equilibrium (LTE) based on planeparallel hydrostatic models of atmospheres (Castelli and Kurucz 2003) using the Kurucz linelists ${ }^{5}$ of atomic and molecular lines. The synthetic spectra of individual stars are summed according to a given mass function. In this paper, the mass function by Chabrier (2005) and the isochrones by Bertelli et al. (2008) and Pietrinferni et al. (2004) (hereinafter: B08 and P04) are used. These models of stellar evolution include the stages of horizontal

\footnotetext{
${ }^{4}$ ftp://ftp.sao.ru/pub/sme/GC4M31/lsf/

${ }^{5}$ http://kurucz.harvard.edu/linelists.html
} 

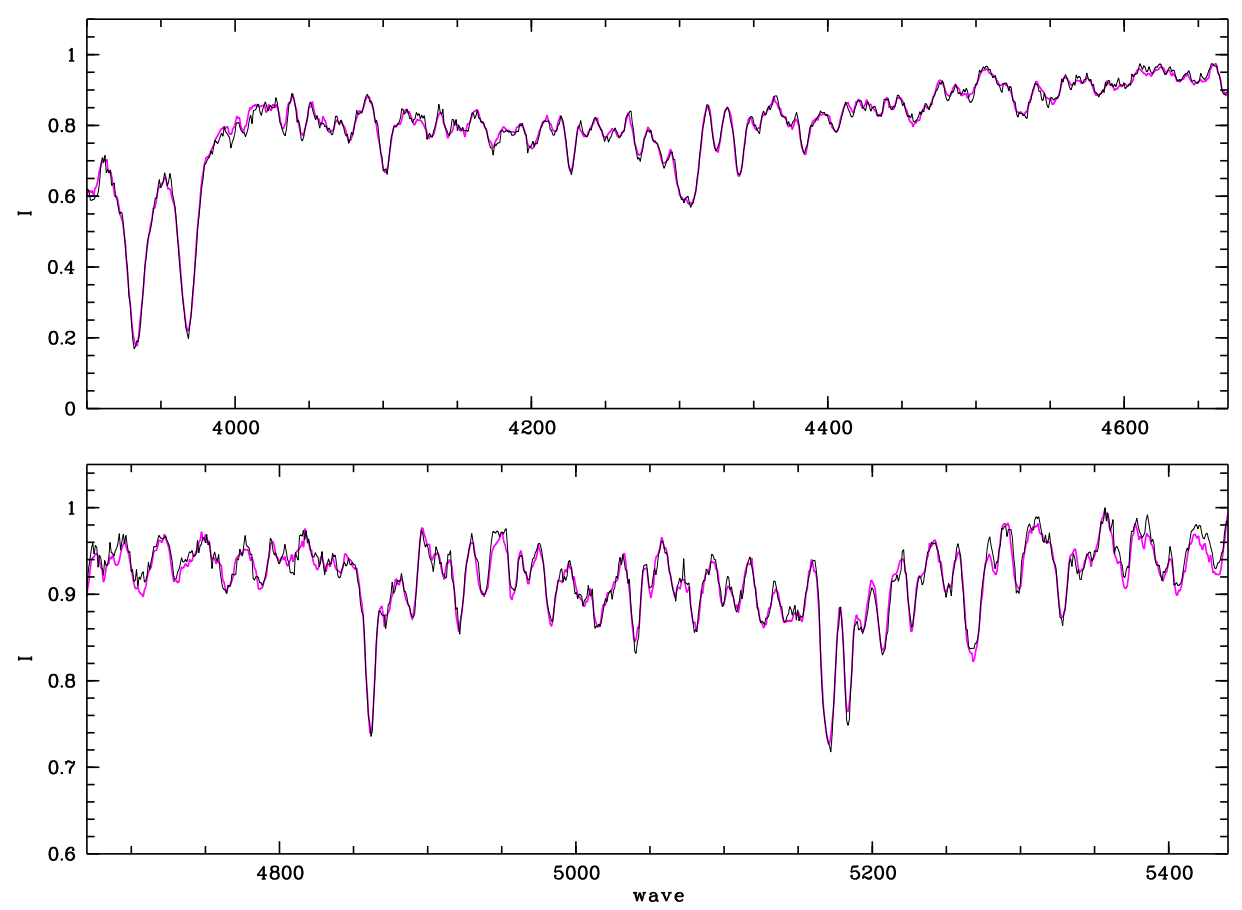

Figure 2. Comparison of the integral spectrum of the Bol 45 cluster with the spectrum of the Bol 50 cluster (pink and black lines, respectively).

Table 3. Results of determination of metallicity and chemical abundances by population synthesis method for the isochrone B08

\begin{tabular}{c|c|c|c|c|c|c|c|c|c}
\hline Object & $\begin{array}{c}\text { Isochrone } \\
(\mathrm{Z}, \mathrm{Y}, \log (\text { Age }))\end{array}$ & $\begin{array}{c}{[\mathrm{Fe} / \mathrm{H}]} \\
(\mathrm{dex})\end{array}$ & $\begin{array}{c}{[\mathrm{C} / \mathrm{Fe}]} \\
(\mathrm{dex})\end{array}$ & $\begin{array}{c}{[\mathrm{N} / \mathrm{Fe}]} \\
(\mathrm{dex})\end{array}$ & $\begin{array}{c}{[\mathrm{Mg} / \mathrm{Fe}]} \\
(\mathrm{dex})\end{array}$ & {$[\mathrm{Ca} / \mathrm{Fe}]$} & {$[\mathrm{Mn} / \mathrm{Fe}]$} & {$[\mathrm{Ti} / \mathrm{Fe}]$} & {$[\mathrm{Cr} / \mathrm{Fe}]$} \\
$(\mathrm{dex})$ & $(\mathrm{dex})$ & $(\mathrm{dex})$ \\
\hline Bol6 & $0.004,0.30,10.05$ & -0.75 & 0.2 & 1.55 & 0.67 & 0.55 & -0.5 & 0.1 & 0.0 \\
\hline Bol45/ & $0.001,0.26,10.05$ & -1.1 & 0.3 & 0.7 & 0.65 & 0.5 & -0.5 & 0.2 & 0.0 \\
Bol50 & $0.002,0.26,10.10$ & & 0.03 & 0.7 & 0.5 & 0.2 & -0.3 & 0.1 & -0.1 \\
\hline Bol20 & $0.002,0.26,10.15$ & -1.0 & 0.05 & 1.4 & 0.5 & 0.38 & -0.5 & 0.2 & 0.0 \\
\hline
\end{tabular}

Table 4. Results of determination of metallicity and chemical abundances by population synthesis method for the isochrone P04

\begin{tabular}{c|c|c|c|c|c|c|c|c|c}
\hline Object & $\begin{array}{c}\text { Isochrone } \\
(\mathrm{Z}, \mathrm{Y}, \mathrm{Age}(\mathrm{Gyr}))\end{array}$ & $\begin{array}{c}{[\mathrm{Fe} / \mathrm{H}]} \\
(\mathrm{dex})\end{array}$ & $\begin{array}{c}{[\mathrm{C} / \mathrm{Fe}]} \\
(\mathrm{dex})\end{array}$ & $\begin{array}{c}{[\mathrm{N} / \mathrm{Fe}]} \\
(\mathrm{dex})\end{array}$ & {$[\mathrm{Mg} / \mathrm{Fe}]$} & {$[\mathrm{Ca} / \mathrm{Fe}]$} & {$[\mathrm{Mn} / \mathrm{Fe}]$} & {$[\mathrm{Ti} / \mathrm{Fe}]$} & {$[\mathrm{Cr} / \mathrm{Fe}]$} \\
$(\mathrm{dex})$ & $(\mathrm{dex})$ & $(\mathrm{dex})$ & $(\mathrm{dex})$ \\
\hline Bol6 & $0.004,0.25,14$ & -0.75 & 0.0 & 1.35 & 0.52 & 0.43 & -0.5 & 0.23 & -0.05 \\
\hline Bol45= & $0.002,0.25,10$ & -1.1 & 0.1 & 0.7 & 0.6 & 0.4 & -0.3 & 0.2 & 0.0 \\
Bol50 & $0.002,0.25,10.5$ & & 0.23 & 0.7 & 0.6 & 0.45 & -0.35 & 0.3 & -0.1 \\
& $0.002,0.25,11$ & & 0.36 & 0.7 & 0.65 & 0.48 & -0.4 & 0.27 & 0.0 \\
\hline Bol20 & $0.002,0.25,12$ & -1.0 & 0.15 & 1.3 & 0.5 & 0.5 & -0.6 & 0.2 & 0.1 \\
\hline
\end{tabular}


(HB) and asymptotic (AGB) branches along with the other main stages of stellar evolution. The ranges of parameter changes in the B08 models are as follows: metallicity ${ }^{6} 0.0001 \leq \mathrm{Z} \leq 0.070$, the logarithm of the age $8.5 \leq \log$ (Age) $\leq 10.15$ in increments of 0.05 and the helium mass fraction $\mathrm{Y}=0.23,0.26,0.30$. The ranges of parameter changes in the P04 models are as follows: metallicity $0.0001 \leq \mathrm{Z} \leq 0.04$ and age 30 Myrs $\leq$ Age $\leq 15$ Gyrs in increments of 0.01 Gyrs for models with Age $<1$ Gyrs and 0.5 Gyrs for models older than 1 Gyrs. The isochrones P04 have a fixed value of the helium mass fraction associated with metallicity $(\mathrm{dY} / \mathrm{dZ} \sim 1.4$ at $\mathrm{Y} \odot=0.2734, \mathrm{Z} \odot=$ 0.0198).

To determine the age, $\mathrm{Y}$, and element abundances, a pixel-by-pixel comparison of the model spectrum and the observed spectrum normalized to the model is performed. According to the shape and depth of the hydrogen lines of the Balmer series, as well as taking into account the balance of the calcium lines Ca I $4227 \AA$ and the lines K and H Ca II $3933.7 \AA$ and $3968.5 \AA$ (the hydrogen line $\mathrm{H} \epsilon$ contributes to the line $\mathrm{H} \mathrm{Ca}$ II), an isochrone is selected. When the metallicity, age, and Y change, the depth of the core and wings of each of the hydrogen lines $\mathrm{H} \delta, \mathrm{H} \gamma$, and $\mathrm{H} \beta$ change differently due to the different contri-

\footnotetext{
${ }^{6}$ The iron abundance in solar units: $[\mathrm{Fe} / \mathrm{H}]=\log \left(N_{F e} / N_{H}\right)-\log \left(N_{F e} / N_{H}\right) \odot, \quad$ where $N_{F e} / N_{H}$ is the ratio of the concentrations of iron and hydrogen by the number of atoms, or by mass. The mass fractions of hydrogen $\mathrm{X}$, helium $\mathrm{Y}$, and metals $\mathrm{Z}$ for the Sun are given in the paper Asplund et al. (2009): $\mathrm{X}+\mathrm{Y}+\mathrm{Z}=1$.
}

butions of stars of different luminosity and spectral classes depending on the wavelength. This fact allows us to determine the metallicity, age, and $\mathrm{Y}$ with sufficient confidence. With this spectral resolution, it is most accurately possible to select the abundances for $\mathrm{Ca}, \mathrm{Mg}$ and $\mathrm{Fe}$, less confidently - for C, N, Mn, Ti and Cr (see Sharina et al. 2013). Typical errors in measuring the abundances are given in the paper Sharina et al. (2020).

\section{RESULTS}

The age, metallicity [Fe/H], Y, and the chemical abundances of the objects under study were determined by the method described in the previous section. Tables 3 and 4 show the results of determining the parameters of the isochrones B08 and P04 used in modeling the spectra, as well as the corresponding abundances of chemical elements. The second column of each of the tables lists the parameters of the isochrones used: metallicity $\mathrm{Z}$, helium mass fraction $\mathrm{Y}$, and age in Gyrs. The $[\mathrm{O} / \mathrm{Fe}]$ content for all objects was taken equal to 0.3 dex, since there are no observational details for it in the investigated spectral range, but it affects the molecular and ionization equilibrium of other elements, in particular, C and $\mathrm{N}$.

The clusters Bol 45 and Bol 50 have almost identical spectra (Fig. 2), so their chemical composition and isochrones used for calculating model spectra are selected the same (Tables 3 


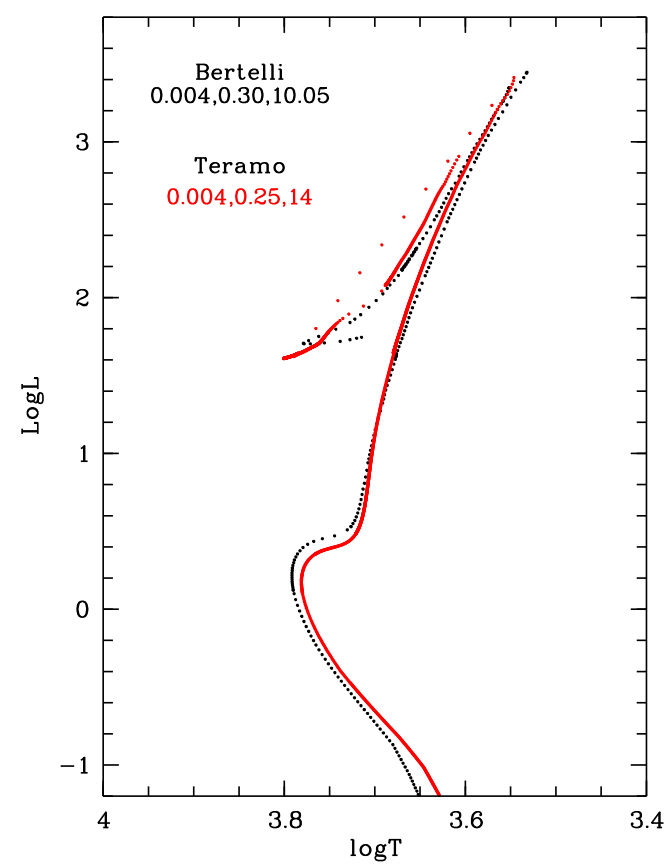

Figure 3. Comparison of the isochrones B08 $(\mathrm{Z}=0.004$, $\mathrm{Y}=0.30, \log ($ Age $)=10.05)$ and $\mathrm{P} 04(\mathrm{Z}=0.004, \mathrm{Y}=0.25$,

Age=14 Gyrs), used to model the Bol 6 spectrum.

and 4). As follows from the tables, the cluster spectrum can be described by different models of stellar evolution. The abundances of chemical elements vary somewhat depending on the choice of the isochrone. For different metallicity, Y, and age,the relative contribution of stars belonging to HB, the MS turnoff, and the tip of the red giant branch (TRGB) change, which affects the depth and shape of atomic and molecular lines. A comparison of the isochrones used to model the Bol 6 spectrum is shown in Fig. 3. The $\mathrm{X}$ and $\mathrm{Y}$ axes are the logarithm of the effective temperature $\mathrm{T}_{\text {eff }}$ in Kelvins and the logarithm of the luminosity L in the solar luminosities, respectively. In the case of Bol 6 (Fig. $3)$, the difference in temperature and luminos- ity at the MS turnoff point for stellar evolution models of stellar evolution that provide the best correspondence between synthetic and observational spectra: $\Delta \mathrm{T}=10^{\log \mathrm{T}_{1}}-10^{\log \mathrm{T}_{2}}=140 \mathrm{~K}$, $\Delta \mathrm{L}=10^{\log \mathrm{L}_{1}}-10^{\log \mathrm{L}_{2}}=0.22 \mathrm{~L} \odot$.

In Fig. 4 and 5 the comparison of model spectra with those observed for Bol 6 in the region of hydrogen lines and lines $\mathrm{K}$ and $\mathrm{H}$ Ca II $3933.7 \AA$ and $3968.5 \AA$ is shown. It can be seen that the model $0.004,0.30,10.05$ is preferable when describing the profile of the $\mathrm{H} \beta$ line, but the isochrone $0.004,0.25,14$ better describes the balance of calcium lines. The uncertainty in the estimation of age and $\mathrm{Y}$ in this case is due to the choice of the theoretical isochrone of stellar evolution for the calculation of a synthetic spectrum. The final choice between isochrones is made based on a comparison of isochrones with the observed distribution of stars on CMD (Section 5.1).

A comparison of the isochrones used to calculate the Bol 45 and Bol 50 synthetic spectra is shown in Fig. 6. The difference in temperature and luminosity for the "blue" end of HB in the isochrones $\mathrm{P} 04$ is $\Delta \mathrm{T}=961 \mathrm{~K}, \Delta \mathrm{L}=3.5 \mathrm{~L} \odot$. The isochrones B08 have the following differences in temperature and luminosity for the "blue" end of HB: $\Delta \mathrm{T}=421 \mathrm{~K}, \Delta \mathrm{L}=3.3 \mathrm{~L} \odot$ and for the stars of the turnoff point: $\Delta \mathrm{T}=291 \mathrm{~K}, \Delta \mathrm{L}=$ $0.17 \mathrm{~L} \odot$. For Bol 45 and Bol 50, all the used models of stellar evolution give almost identical estimates of $[\mathrm{Fe} / \mathrm{H}]$ when comparing the calculated synthetic spectra with the observed ones. How- 


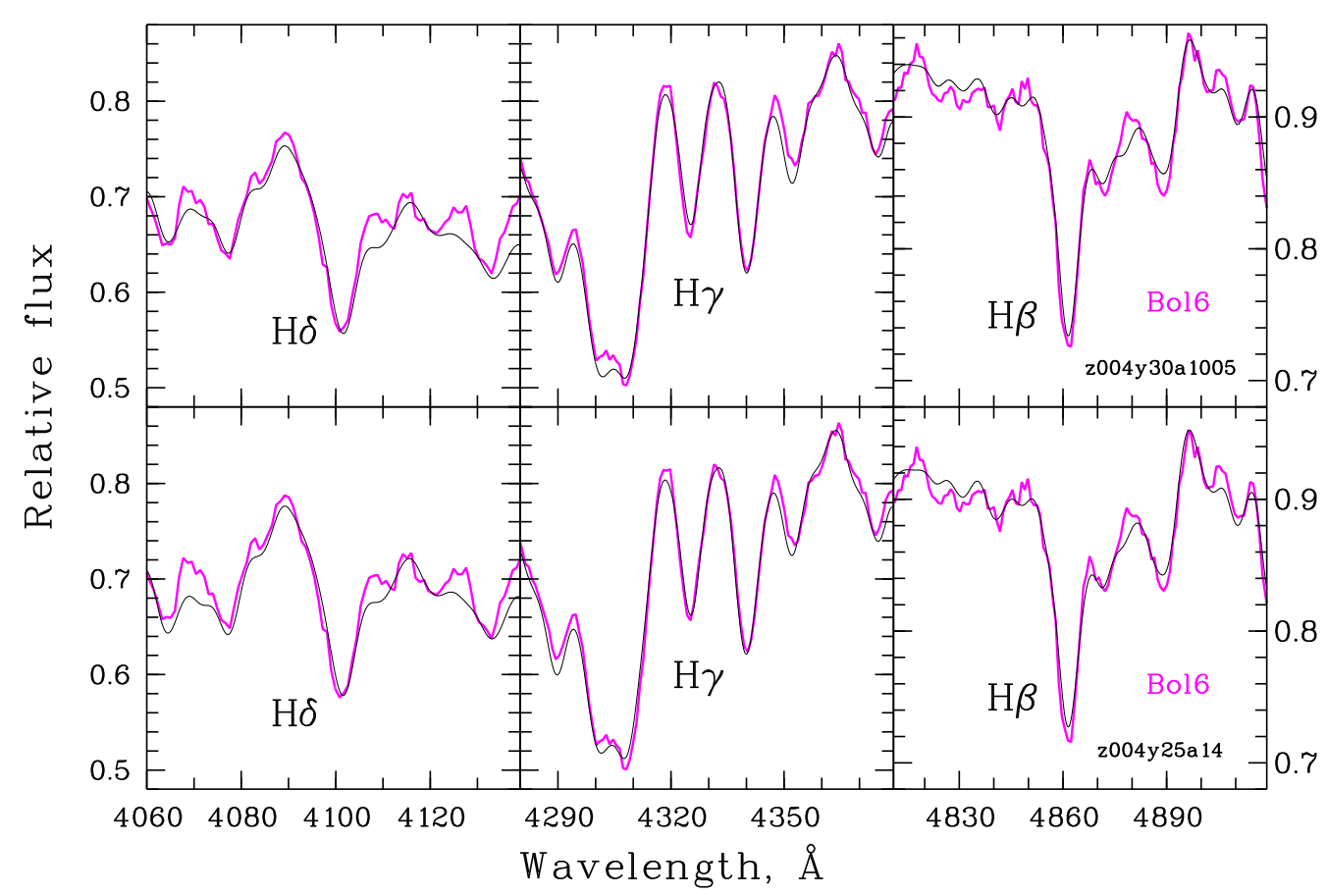

Figure 4. Comparison of the spectrum of the Bol 6 cluster (pink line) with the synthetic one in the region of hydrogen lines. Upper panel - synthetic spectrum calculated with the isochrone $\mathrm{B} 08: \mathrm{Z}=0.004, \mathrm{Y}=0.30, \log ($ Age $)=$ 10.05; lower panel - with the isochrone P04: $\mathrm{Z}=0.004, \mathrm{Y}=0.25$, Age $=14$ Gyrs.

ever, the abundances of other chemical elements differ depending on the choice of the isochrone (Tables 3 and 4). The synthetic spectra (Fig. 7, 8 and 9) calculated with the selected isochrones P04 better describe the hydrogen lines in the observed spectra of Bol 45 and Bol 50 and give close age estimates and a smaller scatter in the determined chemical element abundances. The best fit of the lines is provided by the isochrone $\mathrm{Z}=0.002, \quad \mathrm{Y}=0.25$, Age $=10.5$ Gyrs. The most optimal variant among the isochrones B08 is an isochrone with the parameters $\mathrm{Z}=0.001, \mathrm{Y}=0.26$, $\log ($ Age $)=10.05$.

In the case of Bol 20, the isochrones B08 and P04 selected during spectrum modeling (Fig. 10, 11, 12 and the corresponding data in Tables 3 and 4) allow a good fit of the observed spectra. A comparison between these isochrones (Fig. 10) shows that the differences between them in temperature and luminosity for the "blue" end of HB are $\Delta \mathrm{T}=140 \mathrm{~K}, \Delta \mathrm{L}=2.9 \mathrm{~L} \odot$ and for the $\mathrm{MS}$ turnoff point $-\Delta \mathrm{T}=143 \mathrm{~K}, \Delta \mathrm{L}=0.18 \mathrm{~L} \odot$.

A comparison of the integrated-light spectra of the four clusters under study with each other, as well as their comparison in the region of hydrogen lines, is available via the website ${ }^{7}$. The comparison confirms the conclusions made in this section: Bol 20 has the most intense hydrogen lines compared to other objects. It is older than other clusters in the sample. Bol 6 is the highest-

\footnotetext{
${ }^{7}$ ftp://ftp.sao.ru/pub/sme/GC4M31/
} 


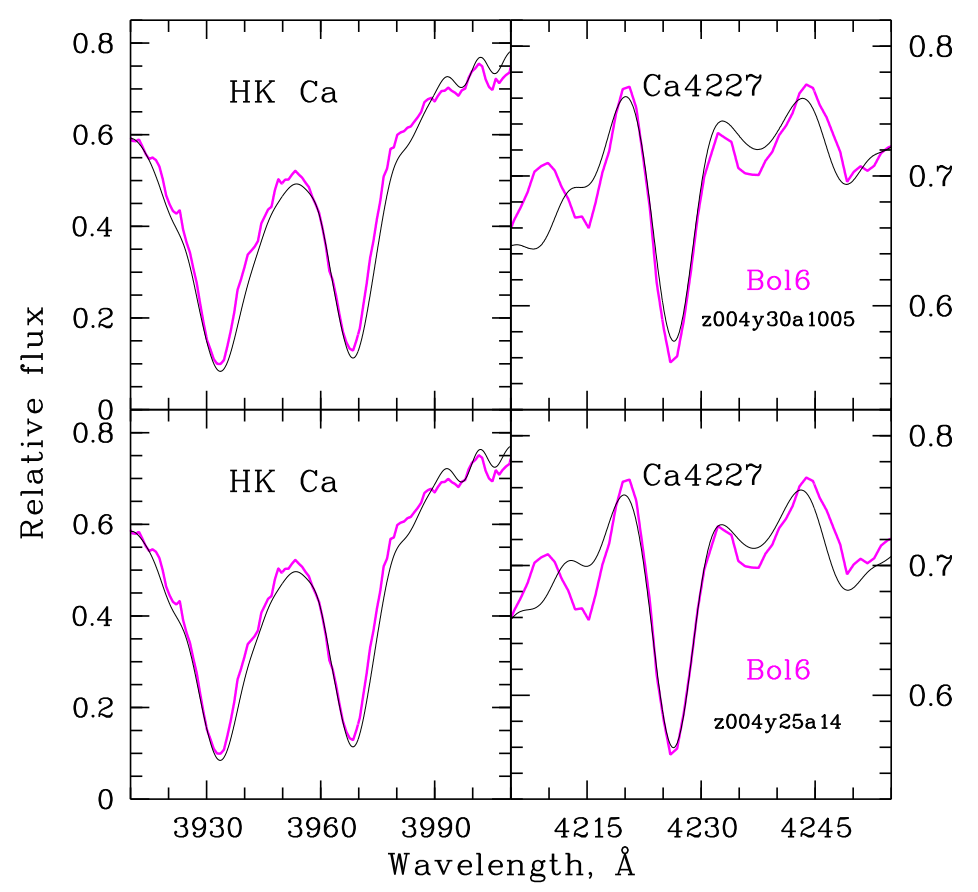

Figure 5. Comparison of the spectrum of the Bol 6 cluster (pink line) with the synthetic one in the region of the lines Ca II K and H and Ca I $4227 \AA$. Upper panel - the synthetic spectrum calculated with the isochrone B08: $\mathrm{Z}=0.004, \mathrm{Y}=0.30, \log ($ Age $)=10.05$; lower panel-with the isochrone $\mathrm{P} 04: \mathrm{Z}=0.004, \mathrm{Y}=0.25$, Age $=14$ Gyrs.

metal object of the sample. It is close to Bol 45 and Bol 50 in age, since the shape and intensity of the hydrogen lines in these three clusters are almost the same.

A comparison of the spectra of the four clusters with the spectra of the globular clusters of the Galaxy from Schiavon et al. (2005), smoothed to the resolution $\mathrm{FWHM}=5.5 \AA$ used in this work, did not reveal objects with completely identical characteristics: age, metallicity and $\mathrm{Y}$. The comparison of the spectra is given on the website ${ }^{8,9}$. The Galaxy clusters with themost similar age, $\mathrm{Y}$ and chemical com-

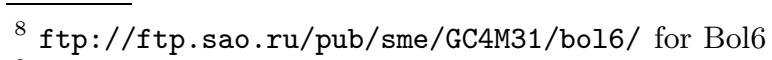

9 ftp://ftp.sao.ru/pub/sme/GC4M31/bol45/ for Bol45 and 50
}

position are: NGC6362 and NGC6652-for Bol 6, NGC6637, NGC6638 and NGC6342-for Bol 45 and Bol 50.

\section{COMPARISON OF THE RESULTS OF SPECTRUM ANALYSIS WITH THE LITERATURE DATA}

Table 5 summarizes the results of this study of the integral spectra of four clusters in M31 and compares it with the literature data. As explained in Section 4, we have adopted for Bol 50 the same estimates of the elemental abundances as for Bol 45. The abundances of the $\alpha$-process elements of the alpha process, given in Table 5, was calculated as the average values of 

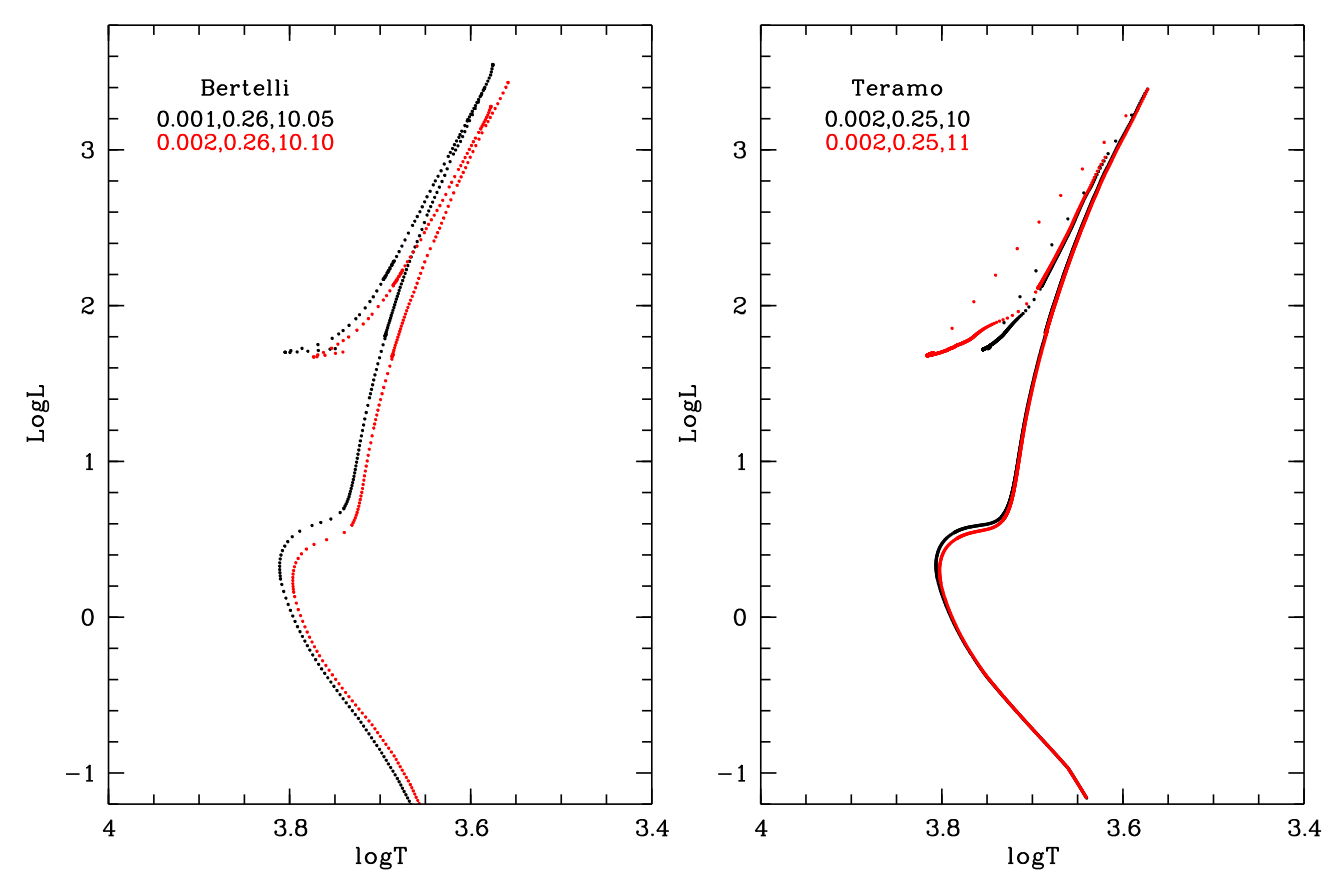

Figure 6. Left panel - comparison of the isochrones B08: $\mathrm{Z}=0.001, \mathrm{Y}=0.26, \log ($ Age $)=10.05$ and $\mathrm{Z}=0.002$, $\mathrm{Y}=0.26, \log ($ Age $)=10.10$. Right panel - comparison of the isochrones $\mathrm{P} 04: \mathrm{Z}=0.002, \mathrm{Y}=0.25$, Age $=10$ Gyrs and $\mathrm{Z}=0.002, \mathrm{Y}=0.25$, Age $=11$ Gyrs, used for calculating the spectra of Bol 45 and Bol 50 .

the abundances of the elements $\mathrm{Mg}, \mathrm{Ca}$ and $\mathrm{O}$. The abundances of the elements of the $\alpha$-process from the works Sakari et al. (2016) and Colucci et al. (2014) obtained by high-resolution spectroscopy are also given there. In these papers, $[\alpha / \mathrm{Fe}]$ is calculated as the average of the abundances of the elements $\mathrm{Ca}$, Si and Ti.

The element abundances, $[\mathrm{Fe} / \mathrm{H}]$ and age (Table 5) determined by us for Bol 6 and Bol 45 are close to the literature values found by highresolution spectroscopy (Colucci et al. 2014; Sakari et al. 2016), with the exception of [C/Fe]. According to the results of this study, both clusters have a higher content of $[\mathrm{C} / \mathrm{Fe}]$ compared to the data of Sakari et al. (2016) from measurements in the infrared range. For Bol 45, we obtained higher $[\mathrm{Mg} / \mathrm{Fe}]$ and $[\mathrm{Ca} / \mathrm{Fe}]$ than in the literature. The reason for the discrepancy in $[\mathrm{C} / \mathrm{Fe}]$ is explained by Sakari et al. (2016). The fact is that the spectral range in the infrared band $\mathrm{H}$ used by these authors is mainly sensitive only to the radiation of stars of the upper branch of red giants, which are characterized by reduced $[\mathrm{C} / \mathrm{Fe}]$ due to changes in the chemical composition during the evolution of stars (see, for example, Sharina and Shimansky (2020) and references therein). The optical spectra contain the radiation of all stars of the clusters. Moreover, the radiation of stars of the MS turnoff point makes a significant contribution to the integrated-light spectrum due to the multiplicity of these objects, according to the 


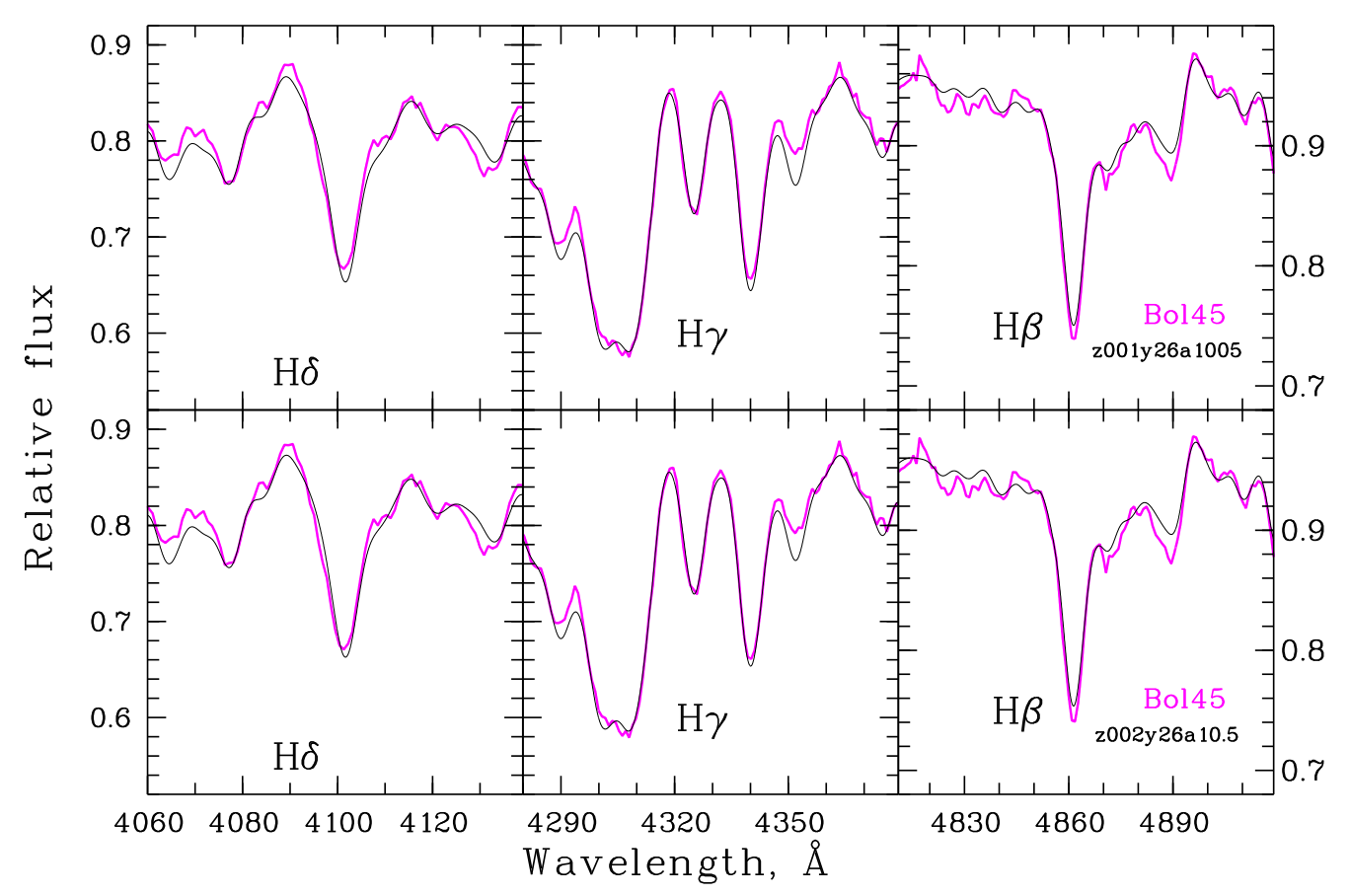

Figure 7. Comparison of the spectrum of the Bol 45 cluster (pink line) with the synthetic one in the region of hydrogen lines. Upper panel - the synthetic spectrum calculated with the isochrone $\mathrm{B} 08: \mathrm{Z}=0.001, \mathrm{Y}=0.26$, $\log ($ Age $)=10.05$; lower panel - with the isochrone P04: $\mathrm{Z}=0.002, \mathrm{Y}=0.25$, Age=10.5 Gyrs.
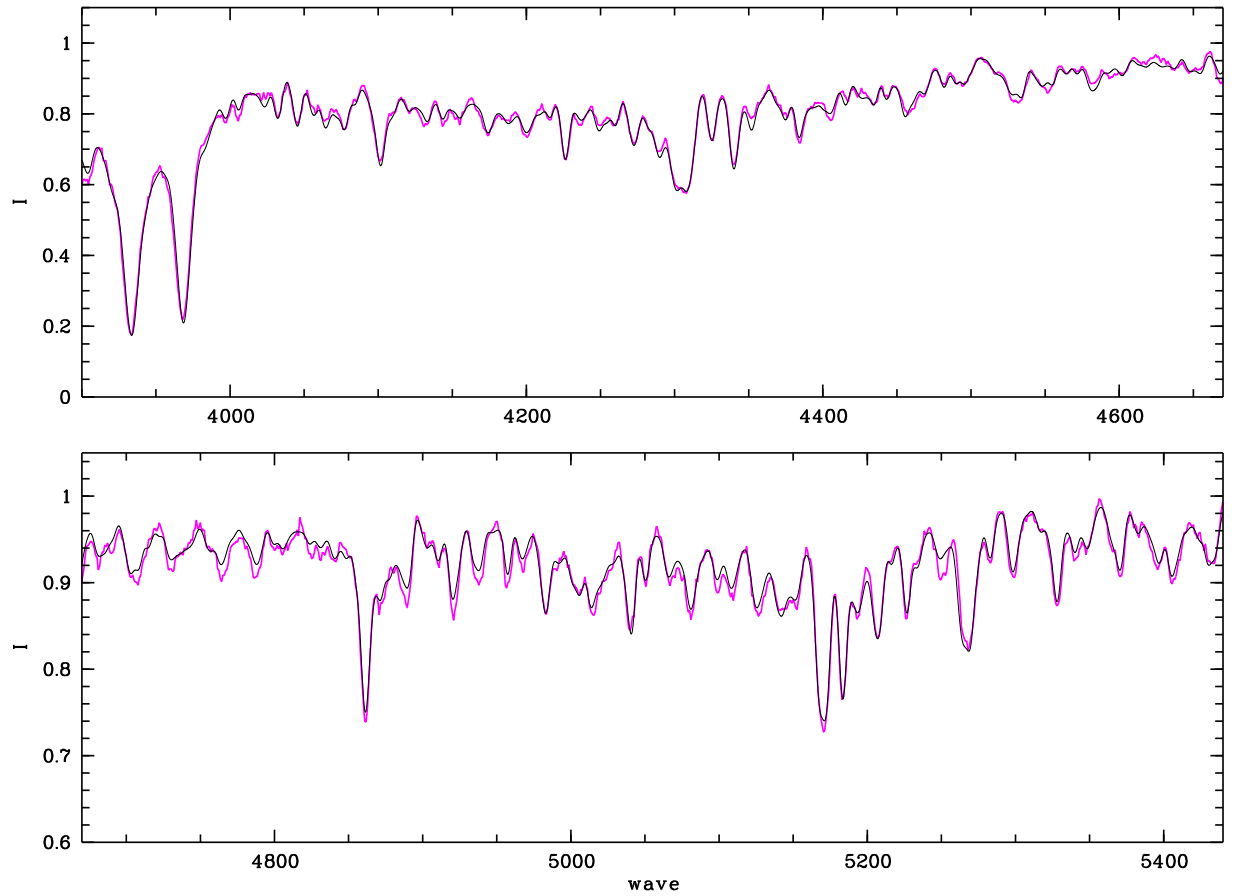

Figure 8. Comparison of the spectrum of the Bol 45 cluster with the synthetic one calculated using the B08 isochrone: $\mathrm{Z}=0.001, \mathrm{Y}=0.26, \log ($ Age $)=10.05$ (pink and black lines, respectively). 

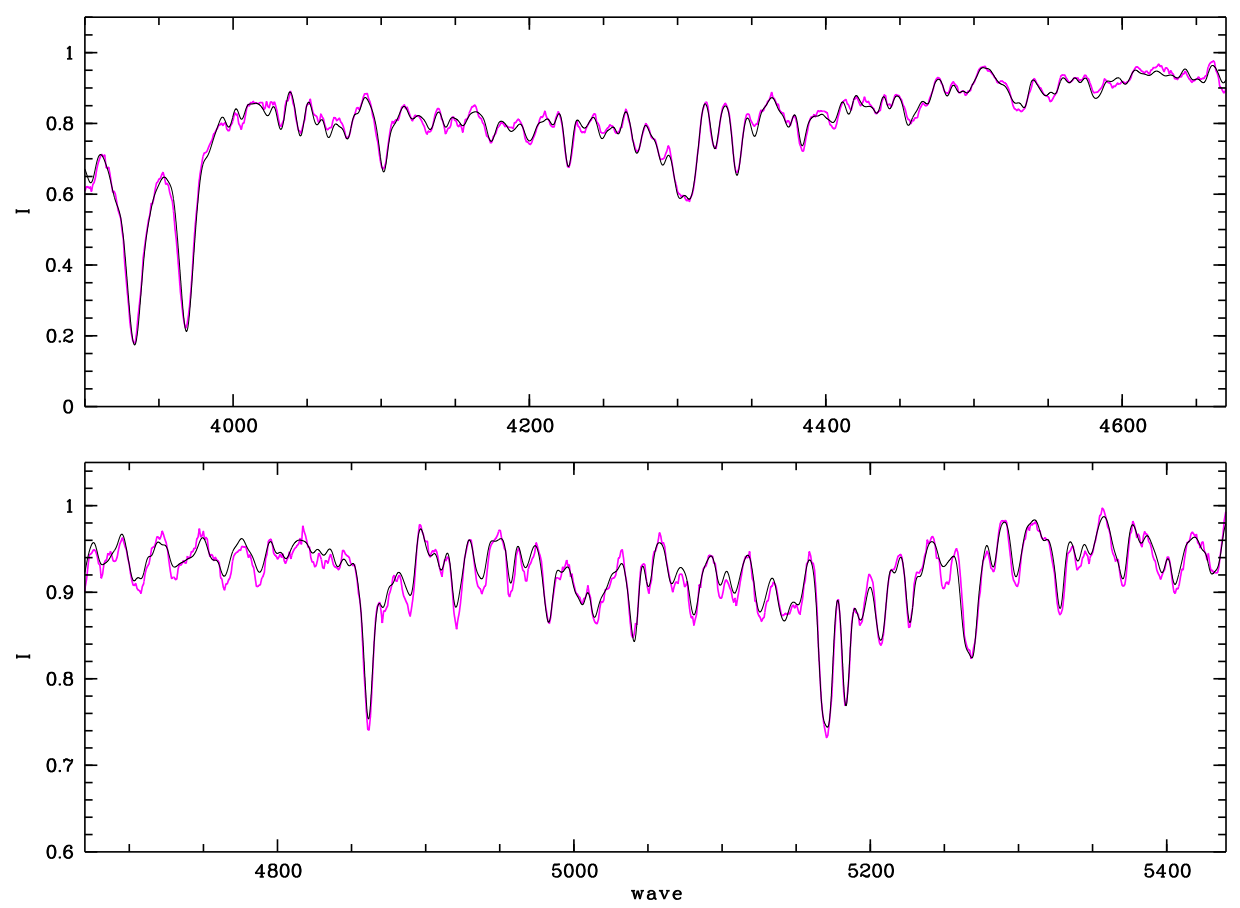

Figure 9. Comparison of the spectrumof the Bol 45 cluster with the synthetic one calculated using the P04 isochrone: $\mathrm{Z}=0.002, \mathrm{Y}=25$, Age $=10.5$ Gyrs. (pink and black lines, respectively).

luminosity function of stars in the cluster (for the contribution of different evolutionary stages to the integrated-light spectrum, see Sharina et al. (2013)). The discrepancies in $[\mathrm{Mg} / \mathrm{Fe}]$ and $[\mathrm{Ca} / \mathrm{Fe}]$ between our and the literature values obtained in studies of high-resolution integratedlight spectra can be explained by insufficient $\mathrm{S} / \mathrm{N}$ in the spectra and differences in the methods used. Differences in the estimates of the abundances in the literature on high-resolution spectra were also noted by Sharina et al. (2020). In the right panels of Fig. 4 in Sharina et al. (2020), it can be seen that the differences between the data of $[\mathrm{Mg} / \mathrm{Fe}]$ and $[\mathrm{Ca} / \mathrm{Fe}]$ Larsen et al. (2017) and Colucci et al. (2017) for some clusters reach the order of 0.3- 0.4 dex. A signif- icant signal has been accumulated in the spectra used in this work. The lines of Mg (Mg I $5183 \AA$ ) and $\mathrm{Ca}$ (Ca I $4227 \AA$ and the lines $\mathrm{K}$ and $\mathrm{H} \mathrm{Ca}$ II $3933.7 \AA$ and $3968.5 \AA$ ), by which the abundances were estimated, are dominant in the spectra at a resolution of FWHM $\sim 5 \AA$. Therefore, the probability of an erroneous determination is low.

For Bol 20 and Bol 50, there are no highresolution spectroscopy results in the literature and, accordingly, $[\mathrm{X} / \mathrm{Fe}]$ for comparison. The metallicity and age obtained by Caldwell et al. (2011) by measuring in the spectra of the Lick indices and comparing them with the model ones are close to our estimates for Bol 50, but significantly different for Bol 20. It should be noted 
Table 5. Comparison of the obtained values of age, $\mathrm{Y},[\mathrm{Fe} / \mathrm{H}]$ and the abundances of chemical elements $[\mathrm{X} / \mathrm{Fe}]$

\begin{tabular}{|c|c|c|c|c|c|c|c|c|c|c|c|c|}
\hline \multicolumn{13}{|l|}{ Obiact } \\
\hline $\begin{array}{c}\text { Object } \\
\text { Ref }\end{array}$ & Ours & $\mathrm{S} 16_{I R}$ & $\mathrm{~S} 16_{o p}$ & C14 & Cl11 & Ours & $\mathrm{S} 16_{I R}$ & C14 Cl11 & Ours & Cl11 & Ours & Cl11 \\
\hline Age & 11.2 & & & 12.5 & & 11 & & 12.5 & 11 & 13.5 & 13 & 7.9 \\
\hline (Gyr) & \pm 1 & & & \pm 2.5 & & \pm 1 & & \pm 2.5 & \pm 1 & \pm 2 & \pm 1 & \pm 2 \\
\hline Y & 0.3 & & & & & 0.26 & & & 0.26 & & 0.26 & \\
\hline$[\mathrm{Fe} / \mathrm{H}]$ & -0.75 & -0.69 & -0.73 & -0.73 & -0.5 & -1.1 & -0.88 & $-0.94 \quad-0.9$ & -1.1 & -0.8 & -1.0 & -0.9 \\
\hline$(\mathrm{dex})$ & \pm 0.1 & \pm 0.05 & \pm 0.02 & \pm 0.1 & \pm 0.1 & \pm 0.1 & \pm 0.07 & $\pm 0.1 \quad \pm 0.1$ & \pm 0.1 & \pm 0.1 & \pm 0.1 & \pm 0.1 \\
\hline$[\mathrm{C} / \mathrm{Fe}]$ & 0.1 & -0.32 & & & & 0.26 & -0.41 & & 0.26 & & 0.1 & \\
\hline$(\operatorname{dex})$ & \pm 0.15 & \pm 0.05 & & & & \pm 0.15 & \pm 0.07 & & \pm 0.15 & & \pm 0.15 & \\
\hline$[\mathrm{N} / \mathrm{Fe}]$ & 1.45 & 1.35 & & & & 0.7 & & & 0.7 & & 1.35 & \\
\hline$(\operatorname{dex})$ & \pm 0.2 & \pm 0.04 & & & & \pm 0.2 & \pm 0.1 & & \pm 0.2 & & \pm 0.2 & \\
\hline$[\mathrm{O} / \mathrm{Fe}]$ & 0.3 & 0.32 & & & & 0.3 & 0.33 & & 0.3 & & 0.3 & \\
\hline$(\operatorname{dex})$ & & \pm 0.04 & & & & & \pm 0.12 & & & & & \\
\hline$[\mathrm{Mg} / \mathrm{Fe}]$ & 0.55 & 0.43 & 0.46 & 0.34 & & 0.6 & 0.22 & 0.04 & 0.6 & & 0.5 & \\
\hline$(\operatorname{dex})$ & \pm 0.1 & \pm 0.05 & \pm 0.1 & \pm 0.03 & & \pm 0.1 & \pm 0.15 & \pm 0.15 & \pm 0.1 & & \pm 0.1 & \\
\hline$[\mathrm{Ca} / \mathrm{Fe}]$ & 0.48 & 0.31 & 0.26 & 0.25 & & 0.45 & 0.2 & 0.22 & 0.45 & & 0.45 & \\
\hline$(\operatorname{dex})$ & \pm 0.1 & \pm 0.07 & \pm 0.02 & \pm 0.05 & & \pm 0.2 & \pm 0.13 & \pm 0.04 & \pm 0.2 & & \pm 0.1 & \\
\hline$[M n / F e]$ & -0.5 & & & & & -0.4 & & & -0.4 & & -0.55 & \\
\hline$(\operatorname{dex})$ & \pm 0.2 & & & & & \pm 0.2 & & & \pm 0.2 & & \pm 0.2 & \\
\hline$[\mathrm{Ti} / \mathrm{Fe}]$ & 0.15 & 0.43 & 0.17 & 0.2 & & 0.2 & 0.27 & 0.16 & 0.2 & & 0.2 & \\
\hline$(\operatorname{dex})$ & \pm 0.2 & \pm 0.07 & \pm 0.05 & \pm 0.05 & & \pm 0.2 & \pm 0.14 & \pm 0.06 & \pm 0.2 & & \pm 0.2 & \\
\hline$[\mathrm{Cr} / \mathrm{Fe}]$ & 0.0 & & & & & -0.05 & & & -0.05 & & 0.05 & \\
\hline$(\operatorname{dex})$ & \pm 0.2 & & & & & \pm 0.2 & & & \pm 0.2 & & \pm 0.2 & \\
\hline$[\alpha / F e]$ & 0.44 & 0.37 & 0.3 & 0.3 & & 0.45 & 0.3 & 0.29 & 0.45 & & 0.41 & \\
\hline$(\operatorname{dex})$ & \pm 0.25 & & & & & \pm 0.25 & & & \pm 0.25 & & \pm 0.25 & \\
\hline
\end{tabular}

Our are the values obtained in this work;

S16 ${ }_{I R}$ - Sakari et al. (2016) by high-resolution spectra in the IR range;

S16 ${ }_{o p}$ - Sakari et al. (2016) by high-resolution spectra in the optical range;

C14 - Colucci et al. (2014) by high-resolution spectra in the optical range;

Cl11 - Caldwell et al. (2011) by moderate resolution spectra in the optical range;

that the method used in this work was tested us- is determined with higher accuracy than by the ing the spectra of well-studied globular clusters method of Lick indices (see, for example, Caldof the Galaxy (Sharina et al. 2020). The age well et al. (2011)). 


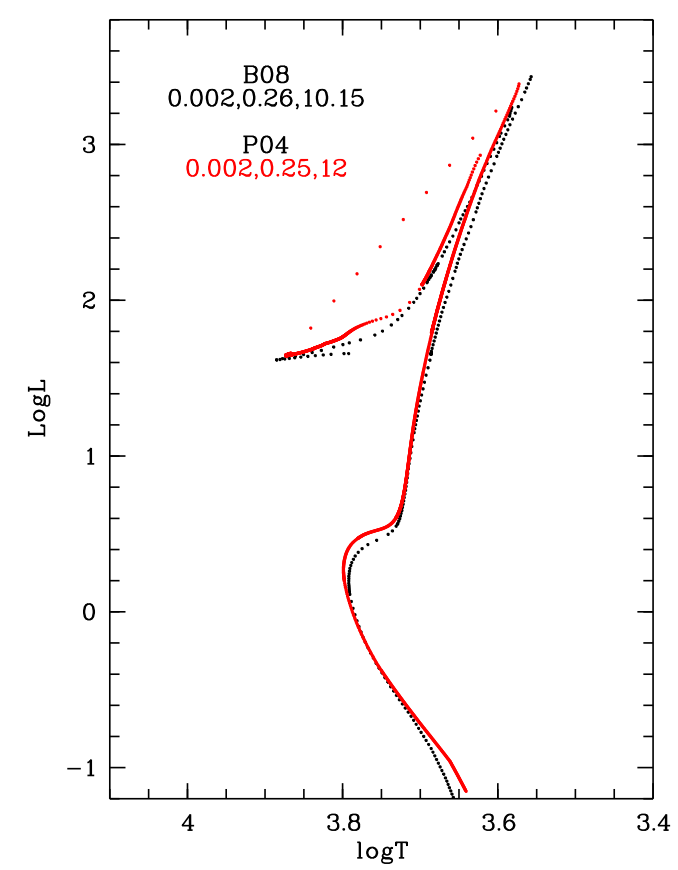

Figure 10. Comparison of the isochrones B08 $(\mathrm{Z}=0.002, \mathrm{Y}=0.26, \log ($ Age $)=10.15)$ and $\mathrm{P} 04$ $(\mathrm{Z}=0.002, \mathrm{Y}=0.25$, Age $=12 \mathrm{Gyrs})$, used to calculate the synthetic spectrum of Bol 20.

\section{1. "Color-Magnitude" Diagrams for Bol 6} and Bol 45 according to Literature Data

For Bol 6 and Bol 45, the literature contains broadband photometry data (Ajhar et al. 1996) obtained at the HST using the WFPC2 camera in the F555W and F814W filters, which can be used to independently verify the results of studying the integrated-light spectra performed in this work.

In Fig. 13 and 14 the comparison of the selected isochrones (Tables 3 and 4) with the observed CMD is shown, based on the literature values of the luminosity level of the horizontal branch VHB (Federici et al. 2012), as well as on the metallicity and age determined by us as a result of the analysis of the spectra (Sections 3 and 4). The depth of the "color- magnitude" diagrams calculated according to photometry data is about $1 \mathrm{~m}$ below the horizontal branch, which allows us to state with caution that the clusters have "red" HBs (Figs. 13 and 14). Background stars contribute to CMD of the studied clusters (Federici et al. 2012). Table 6 shows the metallicity and color excesses $\mathrm{E}(\mathrm{B}-\mathrm{V})$ as well as the distance modules to Bol 6 and Bol 45 obtained as a result of our work with stellar photometry data, in comparison with the corresponding values taken from the literature. In the last column, the method of determining the distance is indicated in parentheses: the luminosity of $\mathrm{HB}$ stars or the luminosity of the top of the red giant branch (TRGB). The level of the horizontal branch of clusters according to data from the literature is also given. It follows from the table that $\mathrm{E}(\mathrm{B}-\mathrm{V})$ and $(\mathrm{m}-\mathrm{M})_{0}$ defined in this paper are close to the literature values. The metallicity of the isochrones used is lower than the literature values obtained by the photometric method, by about 0.06-0.16 dex.

\subsubsection{Determination of the Distance to Clusters by} the Luminosity of the Tip of the Red Giant Branch

The luminosity of TRGB stars is one of the most accurate indicators of the distance to galax- 

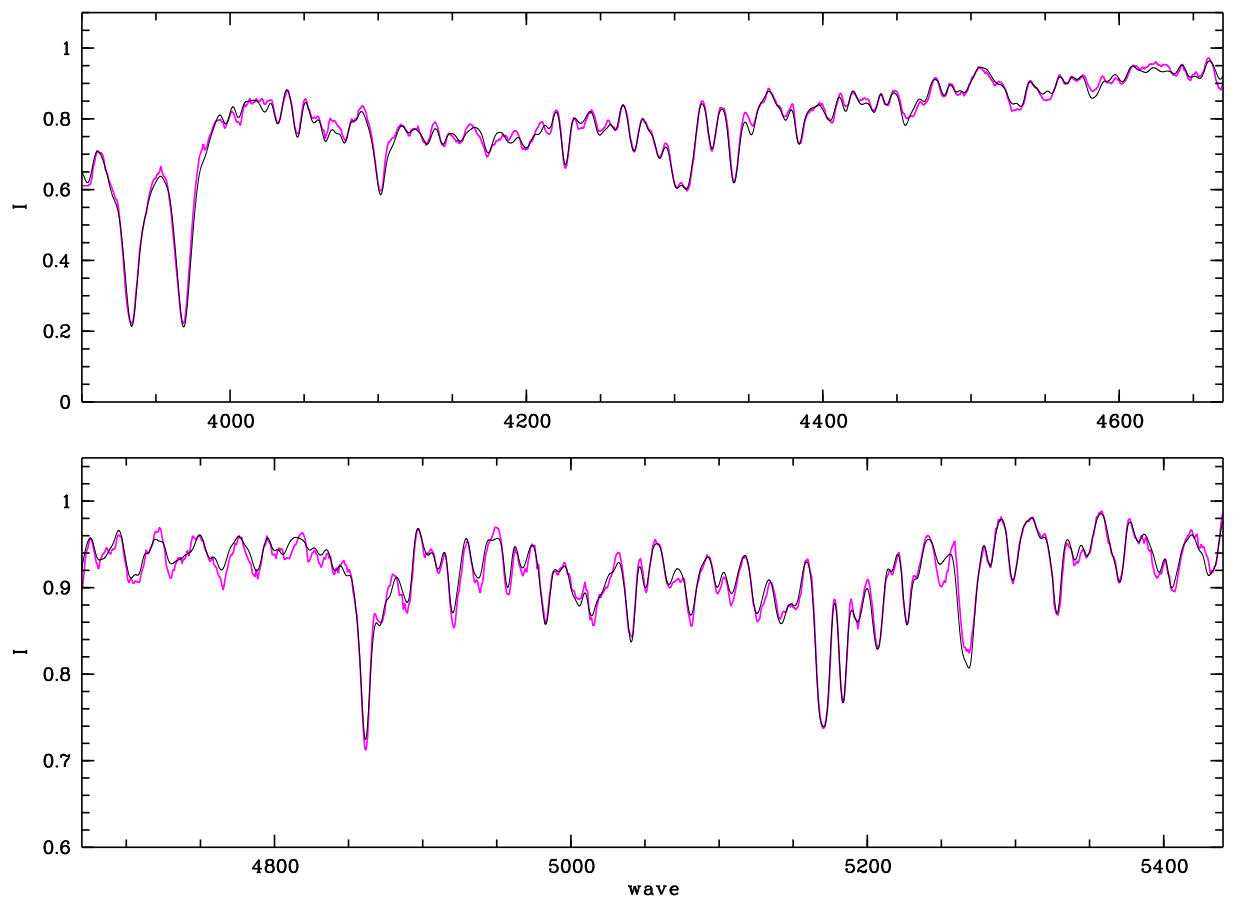

Figure 11. Comparison of the spectrumof the Bol 20 cluster (pink line)with themodel (black line), calculated using the isochrone $\mathrm{B} 08(\mathrm{Z}=0.002, \mathrm{Y}=26, \log ($ Age $)=10.15)$.
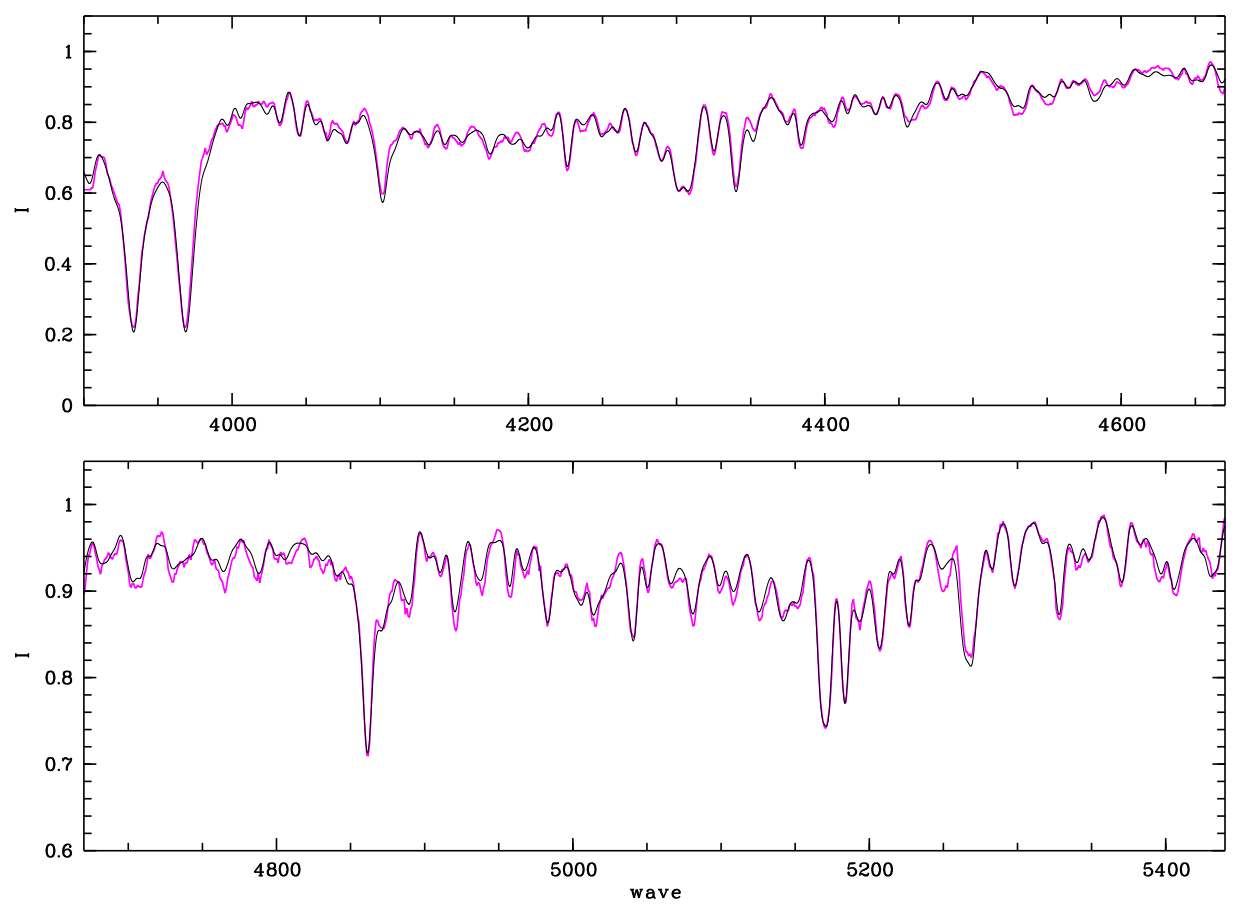

Figure 12. Comparison of the spectrumof the cluster Bol 20 (pink line) and the model (black line), calculated using the isochrone $\mathrm{P} 04(\mathrm{Z}=0.002, \mathrm{Y}=25, \mathrm{Age}=12$ Gyrs $)$. 
ies in which there is an old, metal-poor stellar population (Lee et al. 1993).

Da Costa and Armadroff (1990) calibrated the method using accurate photometry of the globular clusters of the Galaxy, the distances to which were well known by that time. The authors showed that the TRGB luminosity in the I-band of the Johnson-Cousins photometric system varies slightly for stars with metallicities in the range $-2.2<[\mathrm{Fe} / \mathrm{H}]<-0.7$ dex and is $\mathrm{M}_{\mathrm{I}, \mathrm{TRGB}}$ $=-4 \cdot^{m} 05$. Hence, the distance modulus can be defined as follows: $(\mathrm{m}-\mathrm{M})_{0}=\mathrm{m}_{\mathrm{I}, \mathrm{TRGB}}-\mathrm{A}_{I}+$ $4 .^{m} 05$, where $\mathrm{m}_{\mathrm{I}, \mathrm{TRGB}}$ is the observed magnitude of TRGB, and $\mathrm{A}_{\mathrm{I}}$ is the correction for extinction in the I-band.

In this paper, we use the method of determining $\mathrm{m}_{\text {I,TRGB }}$ by Sakai et al. (1996), according to which the luminosity function of red giants was calculated as the sum of Gaussians corresponding to the stellar magnitudes of red giants and normalized for photometric errors in the measurement of stellar magnitudes.

Before calculating the luminosity function, background stars and the AGB stars were removed. The obtained luminosity functions and the corresponding mathematical filters determining TRGB, calculated using the formulae (A1) and (A2) from Sakai et al. (1996) for Bol 6 and Bol 45, are shown in Fig. 15. Using this method, we got the following values: $\mathrm{m}_{\mathrm{I}, \mathrm{TRGB}}=21.0 \pm$ 0.26 for Bol 6 and $\mathrm{m}_{\mathrm{I}, \mathrm{TRGB}}=20.85 \pm 0.21$ for Bol 45. It follows that the distance modules (m$\mathrm{M})_{0}=24.72 \pm 0.26$ for $\mathrm{A}_{\mathrm{I}}=0 \cdot^{m} 17$ for Bol 6 and $24.59 \pm 0.21$ for Bol 45. Comparison of the selected isochrones with the observed CMDs taking into account the obtained distance modules are available online ${ }^{10,11}$. Based on the results of determining the distances by the TRGB luminosity, the distance of clusters from the center of M31 is estimated: $R_{\mathrm{M} 31}=135$ and $85 \mathrm{kpc}$ for Bol 6 and Bol 45, respectively.

\section{DISCUSSION OF THE RESULTS AND CONCLUSIONS}

In this paper, the abundances of the elements C, N, O, Mg, Ca, Mn, Ti and $\mathrm{Cr}$ are determined for the first time for the globular clusters Bol 50 and Bol 20 in the spiral galaxy M31 closest to us. The method used in this work was developed by Sharina et al. (2020). The chemical composition for the other two clusters studied by us in M31 - Bol 6 and Bol 45 - is generally consistent within the error limits with the literature values obtained by high-resolution spectroscopy. For all four objects, the age, metallicity $[\mathrm{Fe} / \mathrm{H}]$ and, for the first time, the helium mass fraction $\mathrm{Y}$ were also determined. The values of the obtained age and metallicity are consistent with the literature data. The results of the study and their comparison with the values available in the literature are given in Table 5. A common feature of the four clusters of the sample is the average metallicity

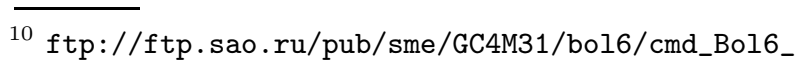
newdist.ps for Bol6

$11 \mathrm{ftp}$ ://ftp.sao.ru/pub/sme/GC4M31/bol45/cmd_ Bol45_newdist.ps for Bol45
} 
Table 6. The results of comparison of the selected isochrones (see Tables 3 and 4) with the observed CMDs and literature values

\begin{tabular}{|c|c|c|c|c|c|}
\hline Object & $\begin{array}{c}{[\mathrm{Fe} / \mathrm{H}]} \\
(\mathrm{dex})\end{array}$ & $\begin{array}{c}\mathrm{E}(\mathrm{B}-\mathrm{V}) \\
\mathrm{mag}\end{array}$ & $\begin{array}{l}\mathrm{V}_{H B} \\
\mathrm{mag}\end{array}$ & $(\mathrm{m}-\mathrm{M})_{0}$ & Reference (Method) \\
\hline \multirow[t]{5}{*}{ Bol6 } & $-0.71 \pm 0.15$ & $0.08 \pm 0.02$ & & $24.57 \pm 0.08$ & $\operatorname{ours}(\mathrm{HB})$ \\
\hline & $-0.75 \pm 0.1$ & $0.17 \pm 0.08$ & & $24.72 \pm 0.26$ & ours(TRGB) \\
\hline & $-0.57 \pm 0.15$ & $0.089 \pm 0.02$ & 25.47 & 24.43 & Ajhar et al. [2](HB) \\
\hline & -0.57 & 0.1 & 25.46 & 24.57 & Rich et al. [37](HB) \\
\hline & -0.55 & 0.08 & 25.46 & 24.56 & Federici et al. $[21](\mathrm{HB})$ \\
\hline \multirow[t]{5}{*}{ Bol45 } & $-1.0 \pm 0.3$ & $0.16 \pm 0.02$ & & $24.51 \pm 0.08$ & ours(HB) \\
\hline & $-1.1 \pm 0.1$ & $0.16 \pm 0.02$ & & $24.59 \pm 0.21$ & ours(TRGB) \\
\hline & $-0.94 \pm 0.27$ & $0.12 \pm 0.03^{B H 82}$ & 25.55 & 24.43 & Ajhar et al. [2](HB) \\
\hline & -0.85 & 0.1 & 25.62 & 24.55 & Rich et al. [37](HB) \\
\hline & -0.9 & 0.16 & 25.62 & 24.55 & Federici et al. [21](HB) \\
\hline
\end{tabular}

BH82 is the value of color excess E(B-V) for Bol 45 from Burstein and Heiles (1982)
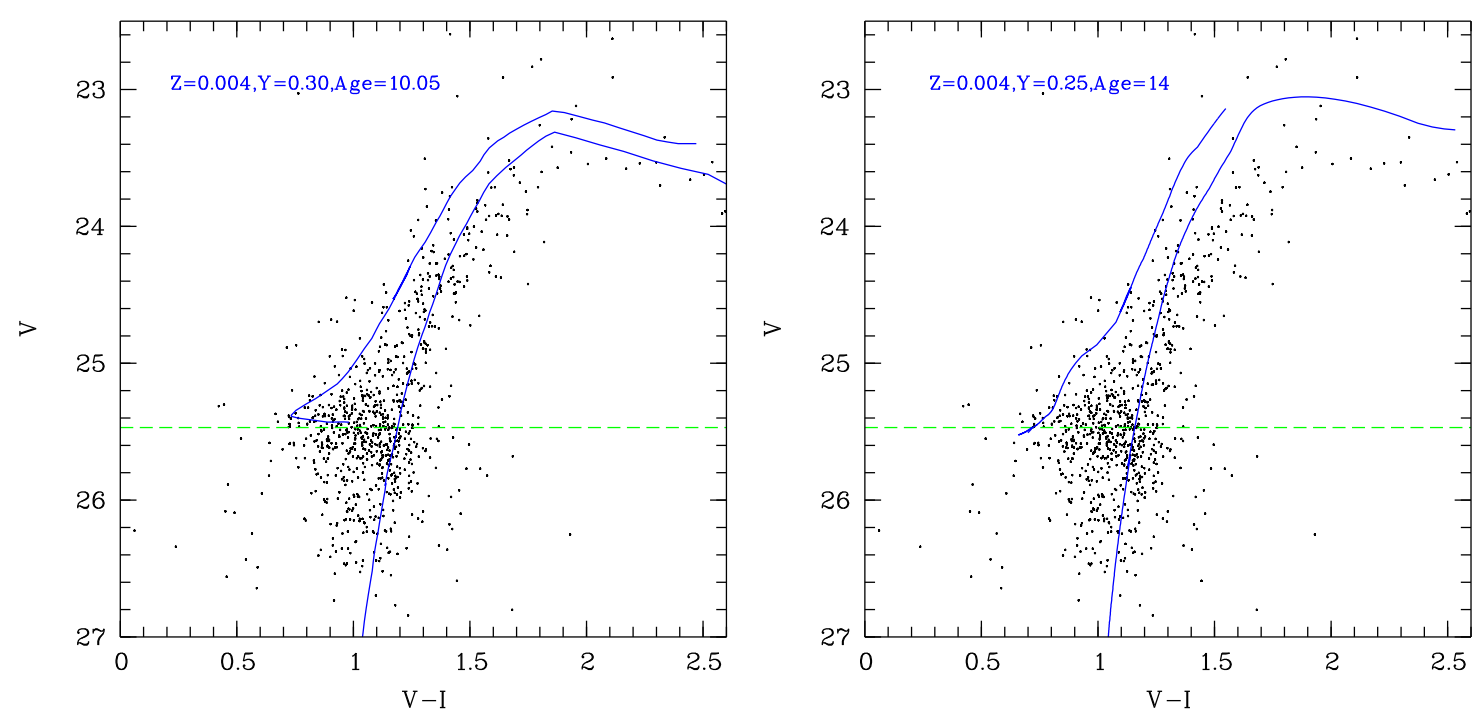

Figure 13. (Left panel- comparison of the Bol 6 CMD with the isochrones B08, $\mathrm{E}(\mathrm{B}-\mathrm{V})=0.08,(\mathrm{~m}-\mathrm{M})_{0}=24.57$. Right panel- comparison of the Bol $6 \mathrm{CMD}$ with the isochrones $\mathrm{P} 04, \mathrm{E}(\mathrm{B}-\mathrm{V})=0.08,(\mathrm{~m}-\mathrm{M})_{0}=24.51$. The green dashed line on both panels shows the level $\mathrm{V}_{H B}$ (Federici et al. 2012).

of $[\mathrm{Fe} / \mathrm{H}][-1.1 ;-0.75]$ dex and the large age of 11 in the models of the chemical evolution of the - 14 Gyrs (see Table 5). Galaxy under the influence of type II superThe obtained abundances correspond to those novae (SNe II) and hypernovae (Kobayashi et 

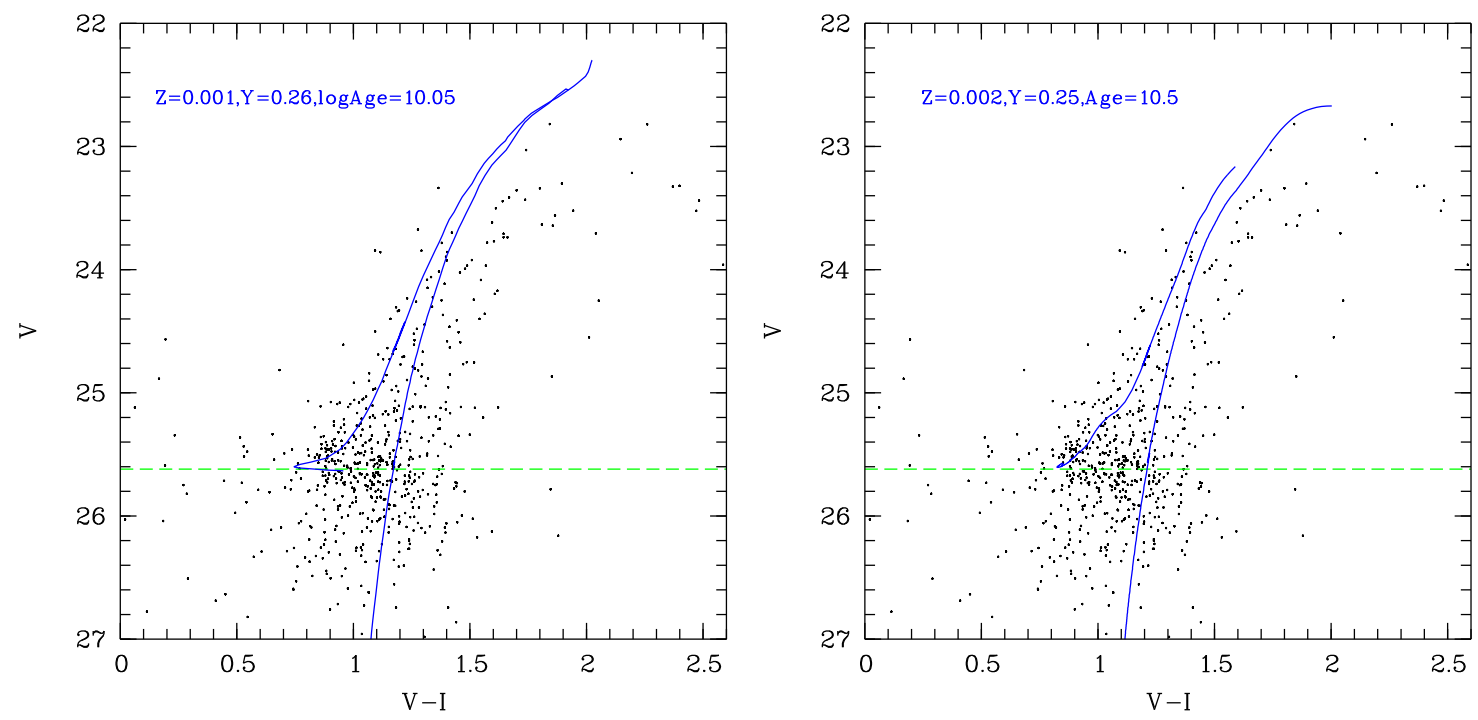

Figure 14. Left panel- comparison of the Bol $45 \mathrm{CMD}$ with the isochrones $\mathrm{B} 08, \mathrm{E}(\mathrm{B}-\mathrm{V})=0.16,(\mathrm{~m}-\mathrm{M})_{0}=24.51$. Right panel- comparison of the Bol $45 \mathrm{CMD}$ with the isochrones $\mathrm{P} 04, \mathrm{E}(\mathrm{B}-\mathrm{V})=0.16,(\mathrm{~m}-\mathrm{M})_{0}=24.51$. The green dashed line on both panels shows the level $\mathrm{V}_{H B}$ (Federici et al. 2012).
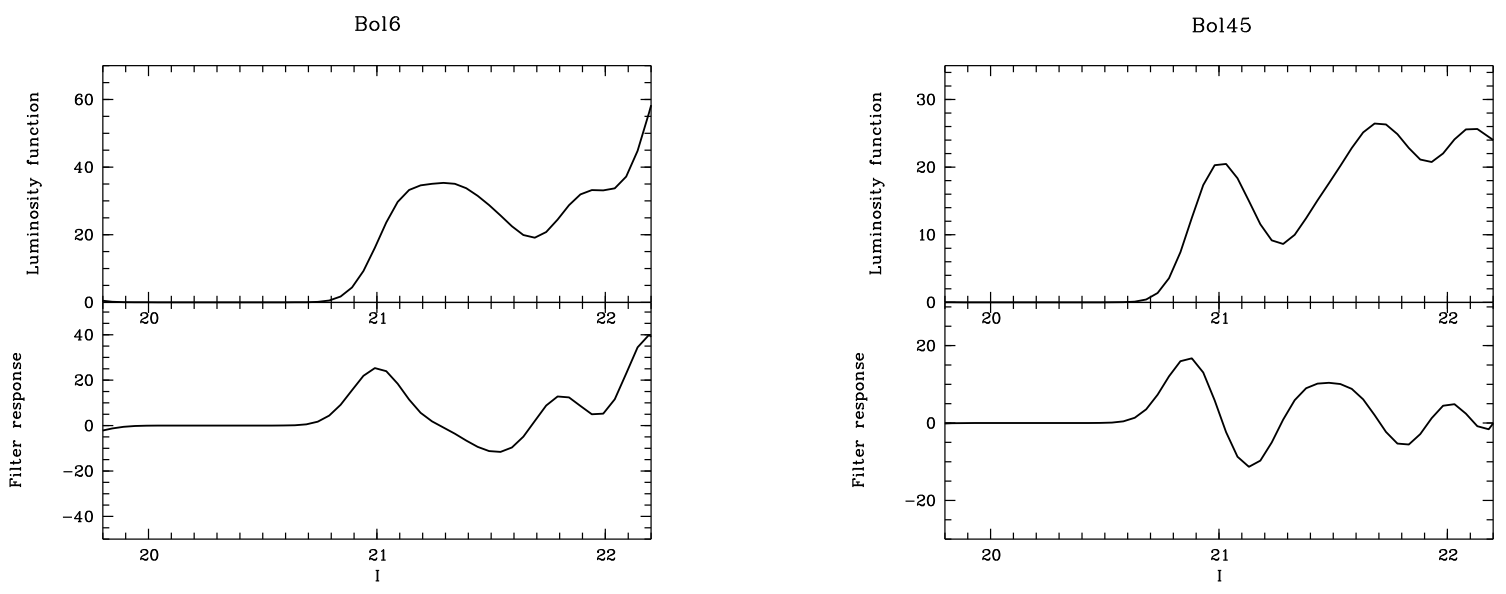

Figure 15. Left panel- the luminosity function of Bol 6 (top) and the result of filtering the luminosity function (bottom), the resulting value is $\mathrm{m}_{I, T R G B}=21.0 \pm 0.26$. Right panel- the luminosity function of Bol 45 (top) and the result of filtering the luminosity function (bottom), the resulting value is $\mathrm{m}_{I, T R G B}=20.85 \pm 0.21$. 
al. (2006), see Fig. 32) in the metallicity range $[\mathrm{Fe} / \mathrm{H}]=[-1.1 ;-0.75]$ dex.

The objects of the study have close celestial coordinates. They are spatially located between M31 and its satellite - the dwarf elliptical galaxy NGC205, but their radial velocities are different. Clusters in this region have large differences in radial velocities and metallicity (Caldwell et al. 2016). These authors identified three populations of globular clusters in M31: high-metal, medium-metal $(-1.5<[\mathrm{Fe} / \mathrm{H}]<0.4)$ with a median value of $[\mathrm{Fe} / \mathrm{H}]=-1$ dex and low-metal. These groups have different spatial distribution. Clusters of medium metallicity, which include our objects, are distributed in a wide area up to galactocentric distances $\mathrm{R}_{M 31} \sim 30 \mathrm{kpc}$ and show on average a weak rotation in the direction of rotation of the M31 disk. These objects are characterized by a high degree of concentration to the center of M31 and a significant spread in radial velocities relative to the velocity of M31. The high-metal group $\left(\mathrm{R}_{M 31}<8 \mathrm{kpc}\right)$ has the kinematics of a disk. Lowmetal clusters are distributed almost uniformly in the projection to the sky and are weakly concentrated towards the center of M31.

The four clusters studied in this work are located at a distance of $4.4<\mathrm{R}_{M 31}<7.3 \mathrm{kpc}$ fromthe center of M31 in the projection to the sky. Their metallicity is lower than the average metallicity of the red giants of M31 halo at a given distance from the center of M31 (see Gilbert et al. (2020) and references therein). The average content of alpha elements in the stars of the inner halo of M31 $([\alpha / \mathrm{Fe}]=0.45 \pm 0.09$ dex) is higher than in the stars of the outer halo $([\alpha / \mathrm{Fe}]=0.3 \pm 0.16 \mathrm{dex})$. The obtained values of $[\alpha / \mathrm{Fe}]$ for four objects correspond to the average value of $[\alpha / \mathrm{Fe}]$ of stars of the inner halo at a given distance from the center of M31.

The proximity of the dwarf galaxy NGC205 to M31 ( $\mathrm{D}_{M 31} \sim 42 \mathrm{kpc}$, (McConnachie (2012), Table 2) and a small difference in speeds between the two galaxies (Geha et al. 2006) indicate a likely interaction in the recent past with a massive neighbor (Davidge 2003, 2005; Thilker 2004). The average metallicity of the stars of the red giant branch of $\mathrm{NGC} 205[\mathrm{Fe} / \mathrm{H}]=-$ 0.9 (Mould 1984) has a large variance of values: \pm 0.5 dex. Sharina et al. (2006) determined the age, metallicity, and $[\alpha / \mathrm{Fe}]$ for five clusters in NGC205 from data of the Lick indices. The metallicity of clusters is in the range from $[\mathrm{Z} / \mathrm{H}]$ $\sim-0.6$ to -1.3 dex, which also includes four clusters of this sample (see Table 5). However, the age range of clusters in NGC205 is large: from 4 to 11 Gyrs. Only Bol 6 shows a radial velocity close to the system velocity of NGC205.

Further studies of the age and chemical composition of globular clusters and stars in M31 and in dwarf satellites will clarify the question of the origin of each of the clusters and their belonging to a particular subsystem. 


\section{ACKNOWLEDGMENTS}

The author is grateful to M. E. Sharina for supervising the work and for providing observational data from the 6-m SAO RAS telescope, and to V. V. Shimansky for providing a modified version of the CLUSTER program for spectrum modeling.

The Digitized Sky Surveys were produced at the Space Telescope Science Institute under U.S. Government grant NAGW-2166.

\section{CONFLICT OF INTEREST}

The authors declare no conflict of interest regarding this paper.
1. V. L. Afanasiev and A. V. Moiseev, Astronomy Letters 31, 194 (2005).

2. E. A. Ajhar, C. J. Grillmair, T. R. Lauer, et al., Astron. J. 111, 1110 (1996).

3. M. Asplund, N. Grevesse, A. J. Sauval and P. Scott, Ann. Review Astron. Astrophys. 47, 481 (2009).

4. K. Banse, P. Crane, P. Grosbol, et al., The Messenger 31, 26 (1983).

5. P. Barmby, D. E. McLaughlin, W. E. Harris, et al., Astron. J. 133, 2764 (2007).

6. G. Bertelli, L. Girardi, P. Marigo and E. Nasi, Astron. and Astrophys. 484, 815 (2008).

7. D. Burstein and C. Heiles, Astron. J. 87, 1165 (1982).

8. N. Caldwell and A. J. Romanowsky, Astrophys. J. 824, 42 (2016).

9. N. Caldwell, P. Harding, H. Morrison, et al., Astron. J. 137, 94 (2009).

10. N. Caldwell, R. Schiavon, H. Morrison, et al., Astron. J. 141, 61 (2011).

11. F. Castelli and R. L. Kurucz, IAU Symp. 210, A20 (2003).

12. E. Cezario, P. R. T. Coelho, A. Alves-Brito, et al., Astron. and Astrophys. 549, A60 (2013).
13. G. Chabrier, Astrophys. Space Sci. Library 327, 441 (2005).

14. J. E. Colucci, R. A. Bernstein and J. G. Cohen, Astrophys. J. 797, 116 (2014).

15. J. E. Colucci, R. A. Bernstein and A. McWilliam, Astrophys. J. 834(2), 105 (2017).

16. G. S. Da Costa and T. E. Armadroff, Astron. J. 100, 162 (1990).

17. T. J. Davidge, Astrophys. J. 597, 289 (2003).

18. T. J. Davidge, Astron. J. 130, 2087 (2005).

19. D. Fabricant, R. Fata, J. Roll, et al., Publ. Astron. Soc. Pacific 117, 1411 (2005).

20. Z. Fan, R. de Grijs, B. Chen, et al., Astron. J. 152, 208 (2016).

21. L. Federici, C. Cacciari, M. Bellazzini, et al., Astron. and Astrophys. 544, 13 (2012).

22. S. Galleti, L. Federici, M. Bellazzini, et al. Astron. and Astrophys. 416, 917 (2004).

23. M. Geha, P. Guhathakurta, R. M. Rich and M. C. Cooper, Astron. J. 131, 332 (2006b).

24. K. M. Gilbert, J. Wojno, E. N. Kirby, et al., Astron. J. 160, 41 (2020).

25. E. Hubble, Astrophys. J. 76, 44 (1932).

26. C. Kobayashi, H. Umeda, K. Nomoto, et al., Astrophys. J. 653, 1145 (2006). 
27. M. Koleva, P. Prugniel, P. Ocvirk, et al., Monthly Notices Royal Astron. Soc. 385, 1998 (2008).

28. M. Koleva, P. Prugniel, A. Bouchard, et al., Astron. and Astrophys. 501, 1269 (2009).

29. S. S. Larsen, J. P. Brodie and J. Strader, å601, A96 (2017).

30. M. G. Lee, W. L. Freedman and B. F. Madore, Astrophys. J. 417, 553 (1993).

31. D. Mackey, G. F. Lewis, B. J. Brewer, et al., Nature (London) 574, 69 (2019).

32. A. W. McConnachie, R. Ibata, N. Martin, et al., Astrophys. J. 686, 36 (2018).

33. A. W. McConnachie, M. J. Irwin, A. M. Ferguson, et al., Monthly Notices Royal Astron. Soc. 356, 979 (2005).

34. A. W. McConnachie, Astron. J. 144, 4 (2012).

35. J. R. Mould, J. Kristian and G. S. Da Costa, Astrophys. J. 278, 575 (1984).

36. A. Pietrinferni, S. Cassisi, M. Salaris and F. Castelli, Astrophys. J. 612, 168 (2004).

37. R. M. Rich, C. E. Corsi, C. Cacciari, et al., Astron. J. 129, 2670 (2005).

38. A. J. Riess, L. M. Macri, S. L. Hoffmann, et al., Astrophys. J. 826, 31 (2016).

39. S. Sakai, B. F. Madore and W. L. Freedman, Astrophys. J. 1996, ApJ, 461, 713 461, 713 (1996).

40. C. M. Sakari, M. D. Shetrone, R. P. Schiavon, et al., Astrophys. J. 829(2), 116 (2016).

41. C. M. Sakari, K. Venn, M. Shetrone, et al., Monthly Notices Royal Astron. Soc. 443, 2285 (2014).

42. R. P. Schiavon, J. A. Rose, S. Courteau and
L. A. MacArthur, Astrophys. J. Suppl. 160(1), 163 (2005).

43. D. J. Schlegel, D. P. Finkbeiner and M. Davis, Astrophys. J. 500, 525 (1998).

44. C. K. Seyfert and J. J. Nassau, Astrophys. J. 102, 377 (1945).

45. M. E. Sharina, V. V. Shimansky and N. N. Shimanskaya, Astrophysical Bulletin 75, 247 (2020).

46. M. E. Sharina, V. V. Shimansky and D. A. Khamidullina, Astrophysical Bulletin 73, 318 (2018).

47. M. E. Sharina, V.V. Shimansky and E. Davoust, Astronomy Reports 57, 410 (2013).

48. M. E. Sharina and V.V. Shimansky,Proceedings of the All-Russian Conference "Ground-Based Astronomy in Russia. 21st Century", Special Astrophysical Observatory of RAS Eds: I.I. Romanyuk, I.A. Yakunin, A.F. Valeev, D.J. Kudryavtsev ,267 (2020).

49. M. E. Sharina, V. L. Afanasiev and T. H. Puzia, Monthly Notices Royal Astron. Soc. 372, 1259 (2006).

50. D. A. Thilker, R. Braun, R. A. M. Walterbos, et al., Astrophys. J. 601, L39 (2004).

51. D. Tody, ASP Conference Series 52, 173 (1993).

52. S. Wang, J. Ma and J. Liu, Astron. and Astrophys. 623, A65 (2019).

53. S. Wang, B. Chen and J. Ma, Astron. and Astrophys. 645, A115 (2020).

Translated by T. Sokolova 